\title{
Germinación y establecimiento de Mimosa aculeaticarpa var. biuncifera (Fabaceae-Mimosoideae)
}

\author{
Seed germination and seedlings establishment of Mimosa aculeaticarpa var. biuncifera \\ (Fabaceae-Mimosoideae)
}

\author{
Numa P. Pavón*, Jesús Ballato-Santos y Claudia Pérez-Pérez \\ Centro de Investigaciones Biológicas. Universidad Autónoma del Estado de Hidalgo. Apartado postal 69-1, Pachuca, Hidalgo, México. \\ *Correspondencia: npavon@uaeh.edu.mx
}

\begin{abstract}
Resumen. El arbusto deciduo espinoso Mimosa aculeaticarpa var. biuncifera que se distribuye en las zonas semiáridas de México tiene potencial para usarse en proyectos de restauración ecológica; sin embargo, no existe información básica que sustente esta afirmación. Evaluar las condiciones óptimas de germinación y establecimiento de la especie fue el objetivo de este estudio. En cuanto a la germinación, se experimentó con 3 factores (escarificación, luz y temperatura) y adicionalmente se determinó la depredación por brúquidos. Para evaluar el establecimiento se midió el efecto de la luz y el nitrógeno en el suelo sobre la sobrevivencia, el crecimiento y la nodulación de las raíces de las plántulas. La escarificación y la temperatura fueron factores significativos en la germinación. Las semillas no fueron fotoblásticas y los parámetros germinativos indicaron que los mejores resultados de germinación se obtuvieron a $30^{\circ}$ C. Las semillas dañadas por los brúquidos no germinaron, la infestación fue de $26.8 \%$ y se definieron 4 especies de brúquidos. La mayor sobrevivencia de plántulas y el mayor crecimiento de las raíces se registraron en condiciones de alta luminosidad. La fertilización del suelo con nitrógeno tuvo efecto negativo significante sobre la sobrevivencia y crecimiento de las plántulas. Estos resultados apoyan la recomendación de usar a M. aculeaticarpa var. biuncifera en proyectos de restauración de zonas semiáridas en México.
\end{abstract}

Palabras clave: brúquidos, fertilización, leguminosas, plántulas, restauración, matorral xerófilo.

\begin{abstract}
Mimosa aculeaticarpa var. biuncifera, spiny drought-deciduous shrub has the potential to be used in restoration projects in degraded semi-arid areas of México. However, basic information that supports this does not exist. The objective of the study was to evaluate the germination conditions and establishment of this species. Germination experiments were realized using 3 factors (scarification, light and temperature). Also, seeds predation for bruquids was registered. We evaluated the effect of light and soil nitrogen on the establishment, for this we considered survival, growth and root nodulation of the shrub seedling. Scarification and temperature were significant dormancybreaking factors. Seeds were not photoblastics and germinative parameters indicated that to $30^{\circ} \mathrm{C}$ the better results were obtained. Seeds damaged by bruquids not germinate; the infestation was $26.8 \%$ and 4 bruquids species were determined. On high brightness conditions, the highest seedling survival and root growth was registered. The nitrogen fertilization of soil had a significant negative effect on survival and growth of the shrub seedling. These results support the recommendation to use $M$. aculeaticarpa var. biuncifera in the restoration projects in degraded semi-arid areas in México.
\end{abstract}

Key words: bruquids, soil fertilization, leguminous plant, seedlings, restoration, xerophilous shrubland.

\section{Introducción}

En México, el género Mimosa está representado por aproximadamente 100 especies distribuidas en las regiones tropicales, subtropicales áridas y semiáridas

Recibido: 05 octubre 2009; aceptado: 12 agosto 2010
(Grether et al., 1996). Las especies que habitan el matorral xerófilo generalmente son arbustos espinosos de gran importancia ecológica, ya que además de formar islas de fertilidad pueden ser nodrizas de diversas especies de cactáceas (Valiente-Banuet y Ezcurra, 1991; Rzedowski y Rzedowski, 2001; Camargo-Ricalde y Dhillion, 2003; Luna-Suárez et al., 2004; Martínez-Pérez et al., 2006). Estas características distinguen a las especies de Mimosa como potenciales para ser usadas en proyectos de restau- 
ración ecológica, ya que generan condiciones ambientales propicias para el establecimiento de otras especies y con ello impulsan la sucesión (Camargo-Ricalde y Grether, 1998; Godínez y Flores-Martínez, 1999; Salamanca, 2000; García-Sánchez, 2005; Martínez-Pérez et al., 2006). Además, varias especies de Mimosa en México tienen uso tradicional, lo que les confiere valor económico y cultural (Camargo-Ricalde et al., 2001), y puede generar mayor aceptación de los programas de restauración ecológica por parte de los pobladores de las comunidades rurales. Sin embargo, el conocimiento sobre la biología de las especies es escaso, por lo que previo a su uso en restauración ecológica es necesario realizar estudios sobre la germinación y establecimiento de las especies de Mimosa, con los que se sustente su potencial.

Las semillas de las especies de Mimosa presentan latencia física, tienen una testa dura impermeable con una capa de cera que resiste la abrasión (van Standen et al., 1989; Camargo-Ricalde y Grether, 1998; Orozco-Almanza et al., 2003; Martínez-Pérez et al., 2006). Las semillas son depredadas por brúquidos que reducen el porcentaje de germinación (Salas et al., 2001; Orozco-Almanza et al., 2003; Romero et al., 2005). El porcentaje de infestación puede ser considerable; por ejemplo, en la especie endémica del suroeste del estado de Tamaulipas M. martindelcampoi se registró una infestación del $40.6 \%$ provocada por 3 especies de brúquidos (Romero et al., 2005). En el caso de algunas especies del género Acacia, se ha observado que los brúquidos producen la escarificación mecánica de las semillas favoreciendo la entrada de agua e incrementando el porcentaje de germinación (Wiegand et al., 1999; Takakura, 2002). Sin embargo, al parecer, esto no ocurre en las especies de Mimosa, ya que la germinación puede reducirse de 90 a $100 \%$ cuando las semillas son depredadas por brúquidos (Orozco-Almanza et al., 2003).

Poco se conoce sobre el establecimiento de plántulas de especies del género Mimosa. Se ha observado en campo que la germinación y el desarrollo de las plántulas de Mimosa tenuiflora ocurren durante la época de lluvias, preferentemente en sitios expuestos a la luz (Camargo y Grether, 1998). La capacidad de nodulación bacteriana y la formación de micorrizas permiten el establecimiento de plántulas en sitios perturbados, sobre suelos infértiles. Incluso, se ha demostrado que el enriquecimiento del suelo con nutrientes, como el fósforo y el nitrógeno, reducen en las plantas la capacidad de interacción con hongos micorrizógenos (Blanke et al., 2005; Camargo-Ricalde et al., 2010), lo que puede ser crítico, dado que las micorrizas tienen un efecto importante en el establecimiento de leguminosas y en particular en la sobrevivencia de algunas especies de Mimosa (García-Sánchez, 2005; Monroy-Ata et al., 2007).
En este trabajo se determinaron las condiciones óptimas de germinación, el efecto de la depredación de semillas por brúquidos y se evaluó el efecto de la luz y la fertilización del suelo sobre el establecimiento de las plántulas, para sustentar la conveniencia de usar la especie Mimosa aculeaticarpa var. biuncifera en programas de restauración ecológica. Se propuso como hipótesis de trabajo que las estrategias de germinación y establecimiento de la especie permitirían soportar las condiciones de alta luminosidad y déficit nutricio que caracterizan a los sitios perturbados de las zonas semiáridas de México.

\section{Materiales y métodos}

Especie en estudio. Para la realización del estudio se seleccionó Mimosa aculeaticarpa var. biuncifera por su capacidad para establecer asociaciones micorrízicas, nodulación bacteriana para fijación biológica de nitrógeno, formar bancos de semillas, amplia cobertura y ser una especie abundante en las zonas semiáridas del centro de México (Puig, 1991; Rzedowski y Rzedowski, 2001; Luna-Suárez et al., 2004; García-Sánchez, 2005). Estos atributos son importantes en la consideración de especies potenciales para restauración ecológica de zonas degradadas (Salamanca, 2000; Meli, 2003).

Mimosa aculeaticarpa var. biuncifera (Benth) Barneby es un arbusto que puede alcanzar hasta $2 \mathrm{~m}$ de altura, tiene hojas con pecíolos de 1 a $3 \mathrm{~cm}$ de largo, con 3 a 9 pares de pinas, sus flores son blancas o blanco-rosáceas, reunidas en cabezuelas axilares de $1 \mathrm{~cm}$ de diámetro; fruto en forma de vaina de color café, en forma linear, curvada o recta, glabra o pubescente (Rzedowski y Rzedowski, 2001). Grether et al. (2006), la consideró como una especie, $M$. biuncifera, cuya distribución abarca las zonas semiáridas de México, desde el norte hasta el centro-sur del país.

Germinación. En enero de 2006, se recolectaron frutos maduros de $M$. aculeaticarpa var. biuncifera de 30 individuos de la población ubicada en el Parque Ecológico

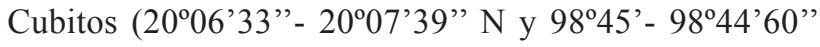
O) en Pachuca, Hidalgo, México. El sitio presenta un clima seco y templado, con una marcada época de lluvias de mayo a octubre (Pavón y Meza-Sánchez, 2009). Los frutos en su mayoría estaban dehiscentes y sin daño evidente. Se obtuvieron aproximadamente 10000 semillas que fueron almacenadas en contenedores de plástico. Durante 2 semanas se recolectaron los brúquidos que salieron de las semillas y estos se fijaron en alcohol al 70 $\%$. Los especímenes fueron determinados por el Dr. Jesús Romero Nápoles (Instituto de Fitosanidad del Colegio 
de Posgraduados, en el Estado de México). El porcentaje de infestación por brúquidos fue determinado de una muestra de 2000 semillas que se clasificaron en sanas y dañadas por brúquidos. Posteriormente, se diseccionaron 200 semillas dañadas y con la ayuda de un estereoscopio se estimó el porcentaje de tejidos internos que fueron consumidos por los brúquidos en cada semilla, incluyendo el embrión.

Se realizaron experimentos factoriales de germinación, usando las semillas sanas (sin evidencia de daño por brúquidos), mediante un arreglo combinatorio de 3 factores (Reyes, 1999). Se probó el efecto del factor escarificación mecánica con 2 niveles (con y sin escarificación), el factor temperatura con 4 niveles $\left(15^{\circ} \mathrm{C}, 20^{\circ} \mathrm{C}, 25^{\circ} \mathrm{C}\right.$ y $\left.30^{\circ} \mathrm{C}\right)$ y el factor luz con 3 niveles (sin luz, luz continua, y luz 12 hrs/día). La distribución de los tratamientos fue completamente al azar. Para realizar cada tratamiento se usaron 10 cajas de Petri con papel filtro como base, humedecido con agua destilada, y se sembraron 10 semillas en cada caja. Se consideró como germinada toda semilla que tuviera la radícula expuesta (de aproximadamente $1 \mathrm{~mm}$ de largo). Los ensayos se mantuvieron por 8 días y la revisión de la germinación de las semillas fue diaria, ya que en otras especies de Mimosa los porcentajes más altos de germinación se presentan en menos de 10 días (Camargo-Ricalde y Grether, 1998; Godínez-Álvarez y Flores-Martínez, 1999).

La escarificación mecánica de las semillas se realizó cortando parte de la testa con un cortaúñas, para evitar dañar el embrión el corte se realizó en el lado opuesto al micrópilo. Los tratamientos se realizaron utilizando una cámara de crecimiento marca VWR (modelo 2015). En los tratamientos con luz se utilizaron 2 lámparas de 40 watts al interior de la cámara. Los tratamientos de luz y obscuridad no se realizaron simultáneamente. El tiempo en la realización entre un tratamiento y otro fue menor a 20 días, por lo que no se consideró el efecto de la edad de las semillas. Para evaluar si el rompimiento de la testa de las semillas, producida por el ataque de los brúquidos, podría tener un efecto (positivo o negativo) sobre la germinación, se repitió el diseño experimental descrito arriba pero usando semillas dañadas por los brúquidos.

Los tratamientos de germinación se analizaron mediante el cálculo de la potencia germinativa, la germinación media, el índice de germinación y la velocidad de germinación (Piedrahita, 1997, 1998; Enríquez et al., 2004; Peña et al., 2004; Weng y Hsu, 2006). Los parámetros germinativos fueron definidos como: Potencia germinativa (PG): porcentaje de germinación total al finalizar el ensayo. Germinación media $\left(\mathrm{G}_{50}\right)$ : número de días transcurridos después de la incubación, para alcanzar el 50\% del porcentaje de germinación final. Se estimó interpolando los 2 días en que se obtuvo el valor más cercano previo y posterior al $50 \%$ de la germinación total.

Índice de germinación $(\mathrm{IG})=$ tiempo de germinación en relación con la capacidad germinativa

$$
I G=\frac{\sum\left(n_{i} t_{i}\right)}{N}
$$

donde: $n i=$ número de semillas germinadas en el día $i ; t=$ número de días después de la siembra; $N=$ total de semillas sembradas.

Velocidad de germinación $(\mathrm{M})=$ relación del número de semillas germinadas con el tiempo de germinación

$$
M=\sum \frac{\left(n_{i}\right)}{t}
$$

donde: $n i=$ numero de semillas germinadas en el día; $t=$ tiempo de germinación desde la siembra hasta la germinación de la última semilla.

El efecto de los factores (escarificación, temperatura y luz) sobre el porcentaje de germinación fue evaluado mediante un modelo lineal generalizado (GLIM), usando un análisis de devianza que es similar al análisis de varianza pero transformando los porcentajes en proporciones y considerando una distribución del error tipo binomial. Para el análisis se utilizó una función de ligamiento logit, la cual linearizó las proporciones utilizando el logaritmo natural (Crawley, 1993). Debido a que la transformación de los porcentajes con la raíz del arcoseno no logró normalizar la distribución de los datos, preferimos usar la distribución real de los mismos usando el GLIM. El análisis se realizó usando el programa GenStat 8.1.

Establecimiento de plántulas. Se realizó un experimento factorial de bloques aleatorizados, donde éstos fueron considerados como réplicas. Los factores evaluados fueron: la luz con 2 niveles (sombra y sin sombra) y el suelo con 2 niveles (nativo y nativo enriquecido con nitrógeno). Las variables dependientes fueron la sobrevivencia, la altura y la biomasa (de la raíz y el tallo) de las plántulas. Previo al experimento, se germinaron semillas escarificadas mecánicamente a $30^{\circ} \mathrm{C}$ y 12 horas de luz por día, dentro de una cámara de crecimiento (VWR). En total se sembraron 528 semillas germinadas, que fueron repartidas equitativamente en 8 almácigos de $60 \times 40 \mathrm{~cm}$ y de $15 \mathrm{~cm}$ de profundidad. Cada almácigo fue considerado como un bloque. Para cada tratamiento de luz se tuvieron 2 almácigos con suelo nativo y 2 con suelo enriquecido con nitrógeno, cada uno con 66 plántulas. 
Los experimentos se mantuvieron durante 14 semanas bajo condiciones de invernadero, donde la humedad relativa fluctuó alrededor del $50 \%$ y la temperatura promedio fue de $25^{\circ} \mathrm{C}$. En cada almácigo se colocó un sensor de yeso, el cual se conectaba diariamente a un medidor watermark, lo que permitió mantener el suelo húmedo entre valores de 20 y 30 centibares. Las condiciones de sombra se obtuvieron cubriendo los almácigos con malla de mosquitero negra teniendo en promedio 323.5 lux (E.S. =96.5); la condición sin sombra, que trató de semejar condiciones abiertas, sin presencia del dosel vegetal, tuvo 7043.3 lux (E.S.= 571). El suelo nativo se recolectó del sitio donde se establece la población de plantas en el Parque Ecológico Cubitos, cuyo $\mathrm{pH}$ es neutro o ligeramente alcalino (6.8 a 7.3) y la materia orgánica es baja (1 a $2.5 \%$ ), lo que indicó deficiencia en nitrógeno. El suelo enriquecido con nitrógeno se preparó agregando sulfato de amonio al suelo nativo $\left(50 \mathrm{~g} \cdot \mathrm{m}^{2}\right)$.

Durante el experimento, semanalmente se registró el número de plántulas que sobrevivían. Los datos se analizaron mediante el cálculo de la función de sobrevivencia, usando la probabilidad de Kaplan-Meier, la cual genera curvas de sobrevivencia expresadas en proporciones con valores entre 0 y 1 (Lee y Wang, 2003). Se realizó un análisis de sobrevivencia para cada factor por separado. Las diferencias estadísticas entre las curvas fueron evaluadas con la prueba de Wilcoxon modificada por Tarone-Ware (Tarone y Ware, 1977). El efecto de la luz y el tipo de suelo sobre la altura y la biomasa (raíz y tallo) de las plántulas que sobrevivieron hasta el final del experimento, se evaluó mediante análisis de varianza factorial con bloques (Zar, 1994). Debido a que los datos no cumplieron con el supuesto de normalidad y que la transformación logarítmica no los normalizó, se decidió utilizar la transformación de rangos, ya que permite realizar pruebas paramétricas sin que los errores se distribuyan de manera normal (Conover e Iman, 1981). Los análisis estadísticos se realizaron usando el programa Systat 12.0.

\section{Resultados}

Germinación. El porcentaje de semillas infestadas por brúquidos fue en promedio del $26.85 \%$ (E.S.=1.58). Se identificaron 4 especies de brúquidos como depredadores de semillas de Mimosa aculeaticarpa var. biuncifera, 3 pertenecientes al género Acanthoscelides (A. speciosus (Shaeffer), A. mexicanus (Sharp) y A. chiricahuae (Fall)) y 1 especie del género Stator (S. pruinius Horn). Se registró un alto consumo de tejidos internos de las semillas depredadas, con un promedio del $89.7 \%$ de pérdida
(E.S. $=0.96$ ); en todas las semillas observadas había sido consumido el embrión.

La germinación fue epigea y fanerocotilar. Debido a la nula germinación de las semillas dañadas, sólo se presentan los resultados obtenidos con tratamientos en los que se usaron semillas sin daño. El porcentaje final de germinación de las semillas escarificadas fue significativamente mayor que en las que no lo fueron $\left(X^{2}=54.5, \mathrm{P}\right.$ $<0.01$ ). Además, los valores más altos de los diferentes parámetros germinativos se obtuvieron en los tratamientos con semillas escarificadas (Cuadro 1). Las semillas de $M$. aculeaticarpa var. biuncifera no fueron fotoblásticas, ya que no se registraron diferencias significativas en el porcentaje de germinación entre los tratamientos con luz y sin $\operatorname{luz}\left(X^{2}=1.3, \mathrm{P}=0.53\right)$.

La temperatura fue un factor significativo en los porcentajes de germinación $\left(X^{2}=11.9, \mathrm{P}=0.03\right)$. Los valores más bajos se obtuvieron a $15^{\circ} \mathrm{C}$ (Cuadro 1 ) y difirieron significativamente de las otras temperaturas ensayadas $(\mathrm{P}=$ $0.003)$. No hubo diferencias en los resultados obtenidos entre $20^{\circ} \mathrm{C}$ y $25^{\circ} \mathrm{C}(\mathrm{P}=0.179)$. El tratamiento con semillas escarificadas sometidas a $30^{\circ} \mathrm{C}$ y sin luz, tuvo los valores más altos de potencia germinativa, velocidad de germinación, índice de germinación e índice $\mathrm{G}_{50}$ (Cuadro 1). El porcentaje de germinación obtenido a $30^{\circ} \mathrm{C}$ fue significativamente mayor que el de las otras temperaturas ensayadas $(\mathrm{P}=0.004)$. La germinación de las semillas se inició en los primeros 2 días después de la siembra bajo los tratamientos de $25^{\circ} \mathrm{C}$ y $30^{\circ} \mathrm{C}$, mientras que a menores temperaturas el inicio de la germinación fue posterior.

Establecimiento de plántulas. Fue evidente que las plántulas sin sombra tuvieron mayor sobrevivencia que las que crecieron bajo sombra $\left(\chi^{2}=8.275, \mathrm{P}=0.004\right)$ (Fig. 1). Así mismo, la sobrevivencia de las que crecieron en suelo nativo fue mayor que las de suelo enriquecido con nitrógeno $\left(\chi^{2}=7.246, P=0.007\right)$ (Fig. 1). Las plántulas bajo sombra se etiolaron desde la segunda semana, su tallo rápidamente se doblaba y morían. Los cotiledones fueron foliares; en las plántulas bajo sombra perduraron durante todo el experimento, mientras que en las plántulas sin sombra sólo permanecieron hasta la décima semana. En ambos casos el resultado fue independiente del tipo de suelo.

La luz y el tipo de suelo tuvieron efectos significativos sobre la altura y la biomasa del tallo y la raíz de las plántulas. La luz generó plántulas de menor altura pero con mayor biomasa, en comparación con las que crecieron bajo sombra. Las plántulas que crecieron bajo sombra tuvieron una altura promedio de $6.09 \mathrm{~cm}($ E.S. $=0.43)$ y las que crecieron sin sombra de $4.99 \mathrm{~cm}(\mathrm{E} . \mathrm{S} .=0.01)(\mathrm{F}=$ 25.7, $\mathrm{P}=0.000$ ) (Fig. 2). La acumulación de biomasa fue significativamente mayor en las plántulas que crecieron sin sombra, tanto en raíz $(\mathrm{F}=151.6, \mathrm{P}=0.000)$ como en 
Cuadro 1. Valores de los parámetros germinativos obtenidos de los ensayos realizados bajo diferentes combinaciones de temperatura, luz y escarificación en semillas de Mimosa aculeaticarpa var. biuncifera (semillas sin evidencia de daño por brúquidos)

\begin{tabular}{|c|c|c|c|c|c|c|c|c|c|c|c|c|}
\hline \multicolumn{7}{|c|}{$15^{\circ} \mathrm{C}$} & \multicolumn{6}{|c|}{$20^{\circ} \mathrm{C}$} \\
\hline & A & $B$ & C & $D$ & $E$ & $F$ & A & $B$ & $C$ & $D$ & $E$ & $F$ \\
\hline PG & 0 & 0 & 4 & 32 & 0 & 20 & 4 & 46 & 4 & 44 & 6 & 29 \\
\hline M & 0 & 0 & 1.29 & 13.1 & 0 & 6.26 & 1.09 & 13.39 & 2.32 & 43.3 & 2.1 & 14.3 \\
\hline IG & 0 & 0 & 0.64 & 5.82 & 0 & 3.15 & 0.59 & 6.38 & 0.89 & 12.9 & 1.08 & 6.14 \\
\hline \multirow[t]{3}{*}{$\mathrm{G}_{50}$} & $\mathrm{X}$ & $\mathrm{X}$ & 6 & 4.5 & $\mathrm{X}$ & 6.5 & 6.5 & 7.5 & 5.5 & 3.5 & 6.5 & 5.5 \\
\hline & \multicolumn{6}{|c|}{$25^{\circ} \mathrm{C}$} & \multicolumn{6}{|c|}{$30^{\circ} \mathrm{C}$} \\
\hline & $A$ & $B$ & C & $D$ & $E$ & $F$ & $A$ & $B$ & C & $D$ & $E$ & $F$ \\
\hline PG & 6 & 72 & 8 & 37 & 1 & 29 & 5 & 80 & 2 & 65 & 8 & 56 \\
\hline M & 3.84 & 55.1 & 4.1 & 26.2 & 0.63 & 19.2 & 5.3 & 67.2 & 1.06 & 45.6 & 6.59 & 42.8 \\
\hline IG & 1.47 & 19.6 & 1.61 & 9.09 & 0.26 & 6.69 & 1.53 & 21.4 & 0.47 & 15.9 & 2.15 & 13.6 \\
\hline $\mathrm{G}_{50}$ & 5.5 & 4.5 & 5 & 5.5 & 5 & 4.5 & 2.5 & 3.5 & 5 & 4.5 & 3.5 & 3.5 \\
\hline
\end{tabular}

Tratamientos. A, semillas sin escarificar bajo sombra; B, semillas escarificadas bajo sombra; C, semillas sin escarificar bajo luz continua; D, semillas escarificadas bajo luz continua; E, semillas sin escarificar bajo $12 \mathrm{hrs} /$ día de luz y sombra; F, semillas escarificadas bajo 12 hrs/día de luz y sombra. Germinación. X, no se registró germinación durante los primeros 8 días de incubación; PG, potencia germinativa; $M$, velocidad de germinación; $\mathrm{IG}$, índice de germinación; $\mathrm{G}_{50}$, germinación media.

tallo $(\mathrm{F}=114.5, \mathrm{P}=0.000)$. Por otra parte, el suelo nativo generó plántulas más altas $(5.76 \mathrm{~cm}$, E.S. $=0.32)$ que aquellas que crecieron en suelo enriquecido $(5.32 \mathrm{~cm}$, E.S.= 0.16) $(\mathrm{F}=9.1, \mathrm{P}=0.003)$. Además, la biomasa de las plántulas que crecieron en suelo nativo fue mayor tanto en raíz $(\mathrm{F}=12.1, \mathrm{P}=0.001)$ como en tallo $(\mathrm{F}=9.1, \mathrm{P}=0.003)$ (Fig. 2). La interacción entre el factor luz y el factor suelo fue significativa en la biomasa de la raíz $(\mathrm{F}=15.2, \mathrm{P}=0.000)$ y la del tallo $(\mathrm{F}=8.8, \mathrm{P}=0.003)$. El tratamiento sin sombra y en suelo nativo generó las plántulas con mayor biomasa y con un mejor desarrollo de las raíces, ya que fueron las únicas que tuvieron nódulos bacterianos (1.68 nódulos por plántula, E.S. $=0.18)$ y la mayor proporción raíz-tallo $(0.44$ - 0.56) $(\mathrm{F}=2.64, \mathrm{P}=0.105)$.

\section{Discusión}

Germinación. El porcentaje de infestación por brúquidos fue relativamente bajo $(26.8 \%)$ ya que en otras especies del género Mimosa como en M. calcicola, M. lancerata, $M$. luisana, M. polyantha, M. purpusii y M. texana var. filipes el porcentaje varió entre el $30 \%$ y $75 \%$ (Camargo-Ricalde et al., 2004). Al parecer, el haber registrado 4 especies de brúquidos depredando a una misma especie de planta es un fenómeno raro (Romero et al., 2005; Lorea et al., 2006) que podría suponer un proceso competitivo entre los brúquidos por el recurso semilla. En otras especies de Mimosa sólo se han encontrado 2 especies de brúquidos (A. mexicana y S. pruininos) (Camargo-Ricalde et al., 2004). Aunado a lo anterior, se observó la presencia de parasitoides en el $8 \%$ de las semillas dañadas. Las interacciones de competencia y de parasitismo podrían explicar, en parte, el bajo valor de infestación. Estos resultados permiten suponer que, aunque sí ocasionan la muerte de las semillas, en términos de la población el efecto de la depredación por brúquidos no es un factor negativo en la disponibilidad de semillas viables, ya que la relativamente baja infestación permite la generación de un banco de semillas y la recolección de las mismas con fines de producción de plantas en vivero.

Las semillas dañadas no germinaron en ninguno de los tratamientos. En algunas especies se ha registrado que los agujeros hechos por los brúquidos en las semillas incrementan la permeabilidad y elevan la germinabilidad (Wiegand et al., 1999). Sin embargo, este fenómeno no ocurrió en M. aculeaticarpa var. biuncifera, y al parecer tampoco ocurre en otras especies de Mimosa que habitan zonas semiáridas del centro del país, ya que se ha probado que no se mejora la germinación, y que de las semillas con 
(a) - Sombra $\quad-\quad$ Sin sombra

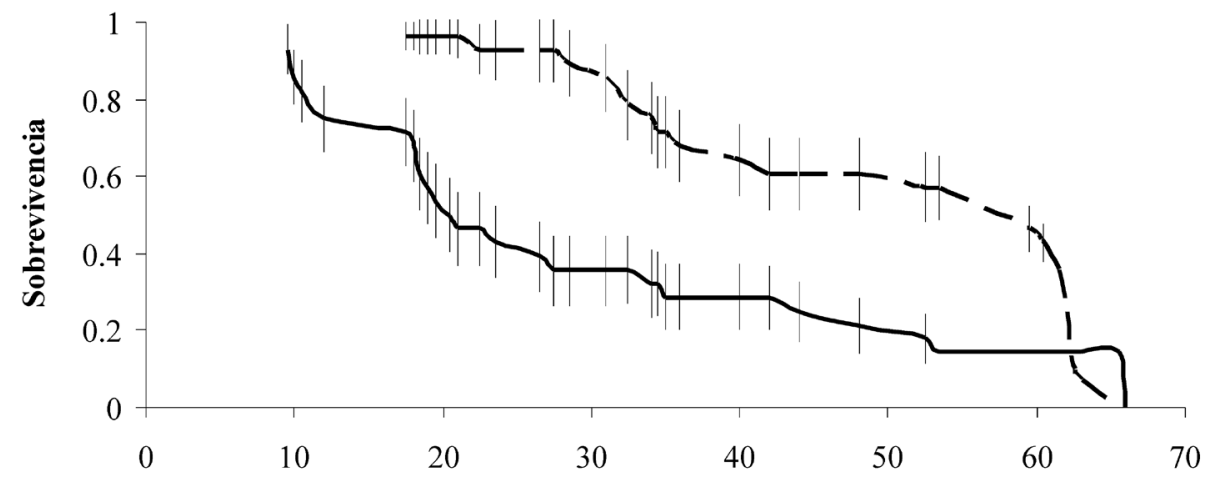

(b) - - Nativo Enriquecido

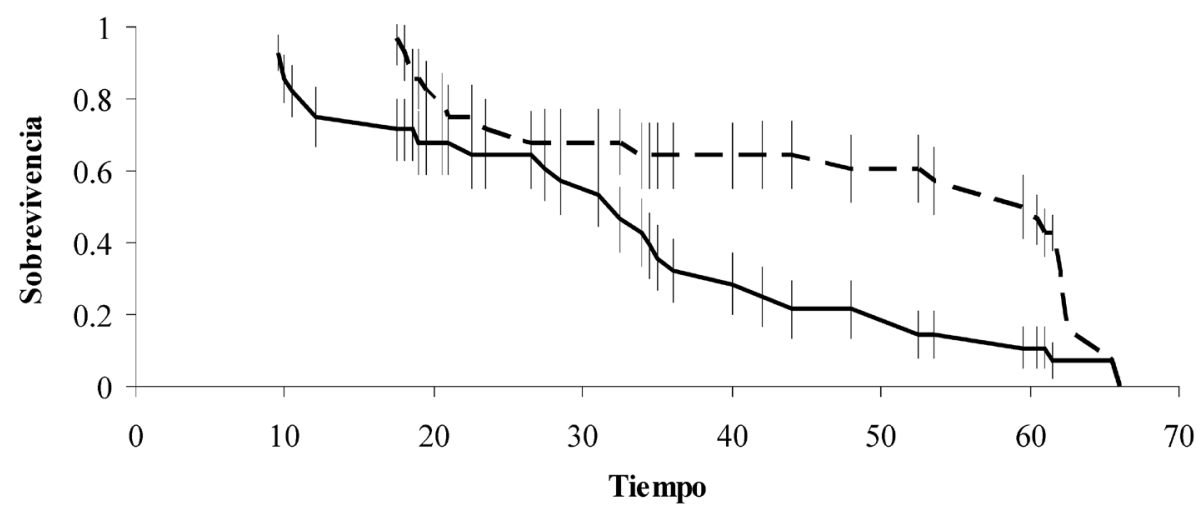

Figura 1. Curvas de proporción de sobrevivencia generadas por la función de probabilidad de sobrevivencia de Kaplan-Meir ( \pm E.S.) de plántulas de Mimosa aculeaticarpa var. biuncifera bajo tratamientos de luz (a) y suelo (b).

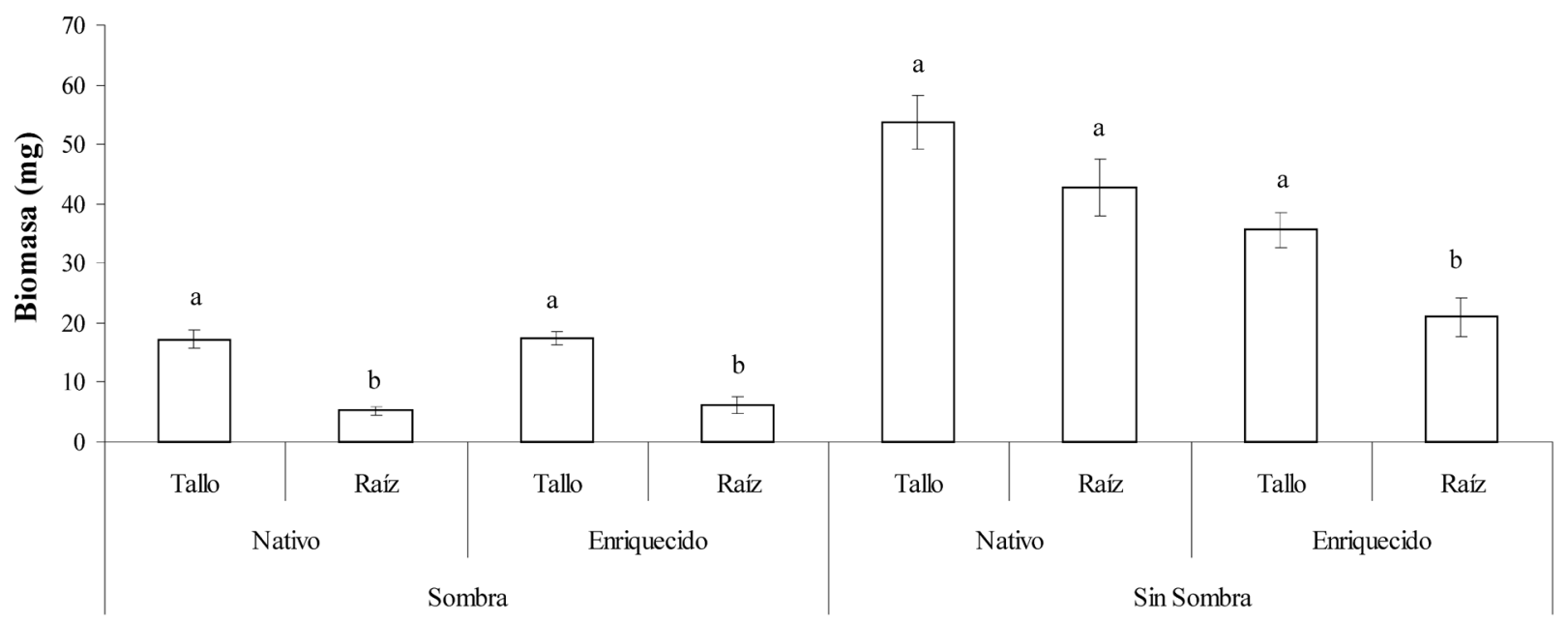

Figura 2. Biomasa promedio ( \pm E.S.) del tallo y las raíces de plántulas de Mimosa aculeaticarpa var. biuncifera después de 14 semanas de crecimiento bajo tratamientos de luz (sombra y sin sombra) y tipos de suelo (nativo y enriquecido). La letras diferentes entre tratamientos indican diferencias significativas $(\mathrm{P}<0.05)$. 
orificio de salida del brúquido sólo germinó del 2-10\% (Orozco-Almanza et al., 2003; Camargo-Ricalde et al., 2004). Lo anterior se debe al daño provocado al embrión y al alto consumo de tejidos internos de las semillas.

En las semillas de leguminosas, la impermeabilidad de la testa está asociada con la desecación de las semillas durante la maduración, lo que sugiere la necesidad de escarificación (Camargo-Ricalde y Grether, 1998). Se probó que la escarificación mecánica es una técnica adecuada para romper la latencia de las semillas, como se ha registrado para otras especies de Mimosa, donde los mejores resultados de germinación se dieron en semillas escarificadas (Camargo y Grether, 1998; Martínez-Pérez et al., 2006). En M. bimucronata, un tratamiento previo de lijado de semillas con un año de antigüedad estimuló la germinación a las $72 \mathrm{hrs,} \mathrm{en} \mathrm{un} 80 \%$ (Vargas-Simón et al., 2003). No se probó la escarificación química, ya que no hay evidencia de que las semillas de esta especie sean tragadas por animales. En forma natural, las semillas de las leguminosas pueden perder su dureza y ser permeables al agua después de la abrasión mecánica por partículas del suelo o después de la descomposición de la testa de las semillas debida a la interacción entre la temperatura y la acción microbiana (Jozef et al., 2003).

Durante la revisión de las semillas que estaban bajo el tratamiento de oscuridad, no se tuvo el cuidado de usar luz verde, lo cual podría haber modificado el porcentaje de germinación debido a la entrada de luz durante las revisiones (Baskin y Baskin, 1998). Sin embargo, las semillas no fueron fotoblásticas, por lo que se estima que el descuido técnico en la revisión de las semillas durante el tratamiento sin luz no produjo un efecto significativo en la germinación. En otras especies de Mimosa no se ha registrado un efecto significativo de la luz, y permaneciendo en el campo las semillas pueden germinar tanto a la sombra del dosel como en sitios desnudos con alta luminosidad (Camargo-Ricalde y Grether, 1998; Orozco-Almanza et al., 2003). Lo anterior tiene una implicación favorable para el uso potencial de la especie en proyectos de restauración de zonas con suelo degradado sin cubierta vegetal. No obstante, como ya se discutirá más adelante, el factor luz sí tuvo gran influencia en la sobrevivencia y desarrollo de las plántulas.

La temperatura fue un factor significativo en la germinación. Los mejores resultados de germinación se obtuvieron en el intervalo que se considera propicio para la germinación de una gran cantidad de especies, el cual fluctúa entre $20^{\circ} \mathrm{C}$ y $35^{\circ} \mathrm{C}$ (Patiño et al., 1983). Sin embargo, en M. luisana las semillas tuvieron un porcentaje de germinación menor a $56 \%$ a una temperatura de $25^{\circ} \mathrm{C}$ (Rivera et al., 2005). En otras especies como M. depauperata y $M$. similis la temperatura óptima de germinación varió entre $20^{\circ}$ y $35^{\circ} \mathrm{C}$, pero en $M$. texana var. texana y M. lancerata tuvieron un intervalo más elevado, fluctuando entre $30^{\circ} \mathrm{y}$ $35^{\circ} \mathrm{C}$ (Orozco-Almanza et al., 2003).

Establecimiento de plántulas. La disminución de la radiación por sombra tuvo un efecto desfavorable en la sobrevivencia de las plántulas, debido a la etiolación. Se sabe que en otras especies de Mimosa, por ejemplo, $M$. tenuiflora, el establecimiento ocurre en áreas abiertas, expuestas a luz solar, por lo que a esas especies se les ha considerado como oportunistas (Camargo-Ricalde y Grether, 1998).

Las plántulas tuvieron un mejor crecimiento de la raíz bajo los tratamientos sin sombra, lo cual es una característica importante para la sobrevivencia durante la sequía, dado que el desarrollo temprano de la raíz posibilita la tolerancia al estrés hídrico, como ha sido registrado en otras especies de ambientes limitados cuyo periodo de establecimiento coincide, en parte, con la sequía intraestival o canícula (Martínez et al., 1994; Padilla, 2008).

La fertilización del suelo con la adición de nitrógeno no tuvo un efecto positivo en las plántulas; por el contrario, la mayor sobrevivencia y biomasa ocurrió en los tratamientos con suelo nativo (con déficit nutricio). Su característica de planta fijadora de nitrógeno le permite sobrevivir en ambientes con suelo limitado en nutrientes, pero cuando se encuentra en suelo con altos niveles de nitrógeno se puede inhibir la iniciación y el tamaño de los nódulos, así como la actividad específica de la fijación (Thomas et al., 2000; Gentili y Huss-Danell, 2002). Es posible que la adición de nutrientes en suelos infértiles modifique procesos de interacción con las raíces de especies de Mimosa. De hecho, recientemente se informó que la adición de fósforo redujo significativamente la colonización por hongos micorrizogenos arbusculares en varias especies del género Mimosa que habitan zonas semiáridas del centro de México (Camargo-Ricalde et al., 2010). En este trabajo, sólo las raíces de plántulas que crecieron sin sombra y en suelo nativo desarrollaron nódulos, lo que, hace evidente que la disponibilidad de nitrógeno en el suelo es un factor que tiene una gran influencia en la fijación simbionte (Gentili y Huss-Danell, 2002). Recientemente, se registró Burkholderia phymatum como una bacteria fijadora capaz de interactuar con especies del género Mimosa; sin embargo, en M. aculeaticarpa la nodulación generada por esta bacteria no fue del todo efectiva (Elliott et al., 2007).

Mimosa aculeaticarpa var. biuncifera es una especie con amplia cobertura, forma banco de semillas, es fijadora de nitrógeno, establece interacciones micorrízicas y tiene una amplia distribución en México, por lo que puede considerarse para proyectos de restauración ecológica de las zonas semiáridas del país. Además, las estrategias de germinación y establecimiento registradas en este trabajo sugieren una alta capacidad para soportar las condiciones 
de alta luminosidad y déficit nutricio que caracterizan a las zonas perturbadas. En particular, la capacidad de fijación de nitrógeno y la formación de micorrizas incrementan su sobrevivencia, por generar una mayor tolerancia al estrés hídrico (Camargo-Ricalde, et al., 2010). Debido a que los resultados indican un efecto negativo en los procesos de interacción entre las raíces y los microorganismos, no se recomienda fertilizar el suelo previo a la siembra. Procede pasar de la teoría a la práctica utilizando la especie en un ensayo donde se registren variables ecofisiológicas que permitan evaluar la respuesta de las plantas de M. aculeaticarpa var. biuncifera durante el proceso de restauración de la cubierta vegetal (Ehleringer y Sandquist, 2006).

\section{Agradecimientos}

Al Dr. Jesús Romero Nápoles, por la determinación de las especies de brúquidos; al Dr. Raúl Ortiz Pulido, por su colaboración en los análisis estadísticos. A Maria Luisa Martínez, Sandra M. Gelviz Gelvez y a los revisores anónimos, por los comentarios y sugerencias. Este trabajo fue apoyado por el proyecto PI-2006/2007 de la Universidad Autónoma del Estado de Hidalgo y el proyecto CONACyT (apoyo complementario SNI, 89992).

\section{Literatura citada}

Baskin, C.C. y J.M. Baskin. 1998. Seeds: ecology, biogeography, and evolution of dormancy and germination. Academic, San Diego. 666 p.

Blanke, V., C. Renker, M. Wagner, K. Fullner, M. Held, A. J. Kuhn y F. Buscot. 2005. Nitrogen supply affects arbuscular mycorrhizal colonization of Artemisia vulgaris in a phosphatepolluted field site. New Phytologist 166:981-992.

Camargo-Ricalde, S. L. y R. Grether. 1998. Germinación, dispersión y establecimiento de plántulas de Mimosa tenuiflora (Leguminosae) en México. Revista de Biología Tropical 46:543-554.

Camargo-Ricalde, S.L., R. Grether, A. Martínez-Bernal, V. GarcíaGarcía y S. Barrios del Rosal. 2001. Especies útiles del género Mimosa (Fabaceae-Mimosoideae) en México. Boletín de la Sociedad Botánica de México 68:33-44.

Camargo-Ricalde, S. L. y S. Dhillion. 2003. Endemic Mimosa species can serve as mycorrhizal "resource islands" within a semiarid communities of the Tehuacán-Cuicatlán, México. Mycorrhiza 13:129-136.

Camargo-Ricalde, S. L., S. Dillion y V. García-García. 2004. Phenology, and seed production and germination of seven endemic Mimosa species (Fabaceae-Mimosoideae) of the Tehuacán-Cuicatlán Valley Mexico. Journal of Arid Environments 58:433-437.

Camargo-Ricalde, S.L., N.M. Montaño, I. Reyes-Jaramillo, C. Jiménez Gonzalez y S. Dhillion. 2010. Effect of mycorrhizae on seedlings of six endemic Mimosa L. species (LeguminosaeMimosoideae) from the semi-arid Tehuacán-Cuicatlán Valley, Mexico. Trees 24:67-78.

Conover, W. J. y R. L. Iman. 1981. Rank transformations as a bridge between parametric and nonparametric statistics. The American Statistician 35:124-129.

Crawley, M. J. 1993. Glim for ecologists. Blackwell scientific publications, Oxford. $379 \mathrm{p}$.

Ehleringer, J.R. y D.R. Sandquist. 2006. Ecophysiological constraints on plant responses in a restoration setting. In Foundations of restoration ecology, D.A. Falk, M.A. Palmer y J.B. Zedler (eds.). Society for ecological restoration international, Island, Washington, D.C. p. 42-58.

Elliott, G. N., W.- M. Chen, J.- H. Chou, H.- C. Wang, S.-Y. Sheu, L. Perin, V. M. Reis, L. Moulin, M. F. Simon, C. Bontemps, J. M. Sutherland, R. Bessi, S. M. de Faria, M. J. Trinick, A. R. Prescott, J. I. Sprent y E. K. James. 2007. Burkholderia phymatum is a highly effective nitrogen fixing symbiont of Mimosa spp. and fixes nitrogen ex planta. New Phytologist 173:168-180.

Enríquez, E., H. Suzán y G. Barrera. 2004. Viabilidad y germinación de semillas de Taxodium mucronatum (Ten.) en el estado de Querétaro, México. México. Agrociencia 38:357-381.

García-Sánchez, R. 2005. Restauración de la cubierta vegetal de los matorrales semiáridos del Valle del Mezquital, Hidalgo, México. www.dama.gov.com; última consulta: 30.IX.2009.

Gentili, F. y K. Huss-Danell. 2002. Phosphorus modifies the effects of nitrogen on nodulation in Split-root systems of Hippophä rhamnoides. New Phytologist 153:53-61.

Godínez, A. H. y A. Flores-Martínez. 1999. Germinación de semillas de 32 especies de plantas de la costa de Guerrero: su utilidad para la restauración ecológica. Polibotánica 11:1-29.

Grether, G. R., S. L. Camargo-Ricalde y A. Martínez-Bernal. 1996. Especies del género Mimosa (Leguminosae) presentes en México. Boletín de la Sociedad Botánica de México 58:149152.

Grether, G.R., A. Martínez-Bernal, M. Luckow y S. Zarate. 2006. Mimosaceae. Flora del valle de Tehuacán-Cuicatlán. Fascículo 44. Instituto de Biología, UNAM, México, D.F. 63 p.

Jozef, A., V. Assche, L. Katrien, A. Debucquoy y A. Rommens. 2003. Seasonal cycles in the germination capacity of buried seeds of some Leguminosae (Fabaceae). New Phytologist 158:315-323.

Lee, E.T. y J. Wang. 2003. Statistical methods for survival data analysis. Wiley-Interscience, New Jersey. 513 p.

Lorea, B. J., J. Romero-Nápoles, C. J. Valdez y J. Carrillo. 2006. Especies y hospederas de los Bruchidae (Insecta Coleoptera) del estado de Jalisco México. Agrociencia 40:511-520. 
Luna-Suárez, S., T. J. Frías-Hernández, V. Olalde-Portugal y L. Dendooven. 2004. Catclaw (Mimosa biuncifera): a pest or a means to restoire soil fertility in heavily eroded soil from central highlands of México? Biology and Fertility of Soils 32:109-113.

Martínez, M. L., P. Moreno-Casasola y E. Rincón. 1994. Sobrevivencia y crecimiento de plántulas en un arbusto endémico de dunas costeras ante condiciones de sequía. Acta Botanica Mexicana 26:53-62.

Martínez-Pérez, G., A. Orozco-Segovia y C. Mantorell. 2006. Efectividad de algunos tratamientos pre-germinativos para ocho especies leñosas de la Mixteca Alta Oaxaqueña con características relevantes para la restauración. Boletín de la Sociedad Botánica de México 79:9-20.

Meli, P. 2003. Restauración ecológica de bosques tropicales, veinte años de investigación académica. Interciencia 28:581-589.

Monroy-Ata, A., J. Estévez-Torres, R. García-Sánchez y R. RíosGómez. 2007. Establecimiento de plantas mediante el uso de micorrizas y de islas de recursos en un matorral xerófilo deteriorado. Boletín de la Sociedad Botánica de México 80 (suplemento):49-57.

Orozco-Almanza, M., L. Ponce de León, R. Grether y E. GarcíaMoya. 2003. Germination of four species of the genus Mimosa (Leguminosae) in a semi-arid zone of Central México. Mexico. Journal of Arid Environments 55:72-92.

Padilla, R. F. 2008. Factores limitantes y estrategias de establecimiento de plántulas leñosas en ambientes semiáridos. Implicaciones para la restauración. Ecosistemas 17:155-159.

Patiño, V. F., P. de la Garza, Y. Villagomez, I. Talavera y F. Camacho. 1983. Guía para la recolección y manejo de semillas forestales. Boletín informativo 62. Instituto Nacional de Investigaciones Forestales, Subsecretaría Forestal, México, D.F. 180 p.

Pavón, N.P. y M. Meza-Sánchez. 2009. Cambio climático en el estado de Hidalgo: clasificación y tendencias climáticas. Universidad Autónoma del Estado de Hidalgo, Pachuca. 168 p.

Peña, E. E., H. Suzán-Azpiri y G. Malda-Barrera. 2004. Viabilidad y germinación de semillas de Taxodium mucronatum (Ten.) en el estado de Querétaro, México. Agrociencia 38:375-381.

Piedrahita, C. E. 1997. Germinación de semillas de Jacaranda copaia bajo condiciones contrastantes de luz. Colombia. Crónica Forestal y del Medio Ambiente 12:1-4.

Piedrahita, C.E. 1998. Aumento del vigoren semillas de Pinus patula (Schlecht. \& Cham.) por el efecto de osmoacondicionamiento. Crónica Forestal y del Medio Ambiente 13:1-21.

Puig, H. 1991. Vegetación de la Huasteca, Mexico. Institut Français de Recherche Scientifique pour le Developpement en Cooperation. ORSTOM / Instituto de Ecología A.C / Centre d'études mexicaines et centraméricaines, CEMCA, México, D.F. 625 p.

Reyes, C. P. 1999. Diseño de experimentos aplicados. Trillas,
México, D.F. 348 p.

Rivera-Aguilar, V., A. H. Godínez, I. C. Manuell-Cacheux y S. Z. Rodríguez. 2005. Physical effects of biological soil crusts on seed germination of two desert plants under laboratory conditions. Journal of Arid Environments 63:344-352.

Romero-Nápoles, J., R. Grether, S.L. Camargo-Ricalde y C. D. Jonson. 2005. Método para la evaluación de daño de semillas por brúquidos (Insecta Coleoptera), en el campo con nuevos registros de hospederos y distribución para el grupo. Entomología Mexicana 4:107-111.

Rzedowski J. y G. Rzedowski. 2001. Flora fanerogámica del Valle de México. Limusa, México, D.F. 1406 p.

Salamanca, B. 2000. Protocolo distrital de restauración ecológica. Convenio DAMA- Fundación Bachaqueros, Bogotá .

Salas, A. D., J. Romero-Nápoles y E. A. García. 2001. Contribución al estudio de los brúquidos (Insecta: Coleoptera) asociadas a fabaceas arbustivas. Acta Universitaria 11:26-32.

Takakura, K. 2002. The specialist seed predator Bruchidius dorsalis (Coleoptera Bruchidae) plays a crucial role in the seed germination of its host plant, Gleditsia japonica (Leguminosae). Functional Ecology 16:252-257.

Tarone, R.E. y J. Ware. 1977. On distribution-free tests for equality of survival distribution. Biometrika 64:156-160.

Thomas R. B., M.A. Bashkin y D. D. Richter. 2000. Nitrogen inhibition of nodulation and $\mathrm{N}_{2}$ fixation of a tropical $\mathrm{N}_{2}$-fixing tree (Gliricidia sepium) grown in elevated atmospheric $\mathrm{CO}_{2}$. New Phytologist 145:233-243.

Valiente-Banuet, A. y E. Ezcurra. 1991. Shade as a cause of the association between the cactus Neobuxbaumia tetetzo and the nurse plant Mimosa luisana in the Tehuacán Valley, México. Journal of Ecology 79:961-971.

van Standen, J., C. J. Manning y K. M. Kelly. 1989. Legumes seeds the structure: function equation. In Advances in legume biology, Monographs on Systematic Botany, vol. 29, C. H. Stirton y J. L. Zarucchi (eds.). Missouri Botanical Garden, St. Louis, Missouri, p. 417-450.

Vargas-Simón, G., F. M. Molina y J. O. Moguer. 2003. Caracterización del fruto, semillas y estudio de germinación del Guapaque (Dialium guianense (AUBL.) Sandwith.) (parte B). Universidad y Ciencia 19:77-83.

Weng, J.-H y F.-H. Hsu. 2006. Variation of germination response to temperature in formosan lily (Lilium formosanum Wall.) collected from different latitudes and elevations in Taiwan. Plant Production Science 9:281-286.

Wiegand, K., F. Jeltsch y D. Ward. 1999. Analysis of population dynamics of Acacia trees in the Negev desert, Israel with a spatially-explicit computer simulation model. Ecological Modelling 117:203-224.

Zar, J.H. 2010. Biostatistical analysis, 5 edición. Prentice Hall, New Jersey. 944 p. 
